\title{
The evaluation and calibration of pressure mapping system for the measurement of the pressure distribution of agricultural tyres
}

by Misiewicz, P.A., Blackburn, K., Richards, T.E., Brighton, J.L. and Godwin, R.J.

Copyright, Publisher and Additional Information: This is the author accepted manuscript. The final published version (version of record) is available online via Elsevier

Please refer to any applicable terms of use of the publisher.

DOI: 10.1016/j.biosystemseng.2014.12.006

Harper Adams

University

Misiewicz, P.A. , Blackburn, K., Richards, T.E., Brighton, J.L. and Godwin, R.J. 2015. The evaluation and calibration of pressure mapping system for the measurement of the pressure distribution of agricultural tyres. Biosystems Engineering. 130, 81-91. 


\section{The evaluation and calibration of pressure mapping system for}

2 the measurement of the pressure distribution of agricultural tyres

4 P. A. Misiewicz ${ }^{\mathrm{a}, 1}$, K. Blackburn ${ }^{\mathrm{a}}$, T. E. Richards ${ }^{\mathrm{a}}$, J. L. Brighton ${ }^{\mathrm{a}}$ and R. J. Godwin ${ }^{\mathrm{a}, 1}$

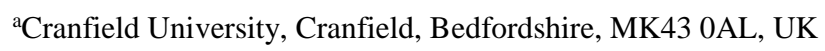

${ }^{1}$ Harper Adams University, Newport, TF10 8NB, UK

Corresponding author: p.misiewicz@ iagre.biz; +44 7752954149

\section{Abstract}

The accuracy of a commercial pressure mapping system was evaluated and a number of techniques for the improvement of pressure measurements were developed. These were required in order to use the pressure mapping system in a tyre/surface interaction study which involved determination of the tyre contact pressure distribution on, both, hard and soil surfaces. In the evaluation of the system, the effect of sensor calibration procedures on the accuracy of the system in measuring pressure was investigated. A purpose built pressure calibration chamber was used to calibrate the sensors, which enabled the proprietary built-in calibration system to be evaluated along with a novel calibration procedure employing, both, an individual and multi-point calibration of each sensing element and the rejection of sensing elements that did not conform to the sensitivity of the majority of the sensing elements. These measures reduced the uncertainty in pressure measurements from $\pm 30 \%$ to $\pm 4 \%$. Further, evaluation of the compliance of the material was also conducted to enable the sensors to be used for interface pressure measurements between two different surface materials other than those used during sensor calibration. As a result, a procedure for normalising the recorded pressure by adjusting the recorded load output to equal the applied load was established. The improvement of the accuracy of the sensors made it possible for the system to be used to determine the pressure distribution resulting from a range of tyres on a hard surface and in the soil profile.

Keywords: pressure mapping system; calibration; contact pressure; soil - tyre interactions. 


\section{Introduction}

Over the last few decades, farm machinery has increased substantially in weight, increasing loads on the soil and exacerbating compaction problems (Horn, Fleige, Peth, \& Peng, 2006). As wheel traffic results in soil compaction (Soane and Ouwerkerk, 1994), a better understanding of soil contact pressure and load transfer to soil through agricultural tyres is essential to provide improved solutions to tyre selection. There is, therefore, a need for an accurate tyre contact pressure measurement system. This article reports on the selection and performance enhancement of a commercial pressure mapping system.

Misiewicz (2010) conducted a review of the commercially available pressure mapping systems, where sensor flexibility, size, pressure resolution, ability to upgradeable the system, customisability, reuse, static vs. dynamic application, test-monitoring capability, modularity and cost were considered. The Tekscan system, I-Scan and Conformat versions (Tekscan, Inc. South Boston, Mass., USA), based on piezo-electric pressure sensors, which enable realtime contact area and pressure distribution to be measured across a multi-sensor array over time (Tekscan, not dated a), was selected for this study due to the sensor size and pressure resolution required to measure the pressure distribution below agricultural tyres. The system measures the load applied to each sensing element and records it as the interface pressure between two surfaces. Tekscan sensors contain thin sensing mats built as a multi-sensor array varying in size, shape, spatial resolution and pressure range. The system contains: (a) piezo-electric pressure sensitive mats (called sensors), (b) data acquisition handle (adaptor) that communicates through a USB interface, (c) data acquisition software and (d) a sensor software map. The system has a wide range of pressure measurement applications including the medical, automotive and furniture design industries. The Tekscan system has an 8-bit output, where each individual sensing element (called a sensel) has a resolution of $0.4 \%$ of the full scale output. The thin construction of the sensors allows them to be deformed and permits minimally intrusive/invasive surface pressure measurements (Tekscan, not dated b).

Before the sensor is used, it should be calibrated to convert its output into engineering units and the output variations between individual sensing elements of any given sensor minimised by applying a uniform pressure across the entire sensor; this process is called equilibration (Tekscan, 2006). There have been a number of studies investigating aspects of the Tekscan system accuracy in determining contact pressure and area of contact (Drewniak, Crisco, Spenciner, \& Fleming, 2007). Sumiya, Suzuki, Kasahara, and Ogata (1998) concluded that 
the Tekscan system does not measure the normal pressures accurately enough for a high level of certainty in terms of absolute values, but it does enable relative comparisons of pressure distribution to be made. Problems of pressure drift, repeatability, linearity and hysteresis were evaluated by Ferguson-Pell, Hagisawa, and Bain (2000) and Wilson, Niosi, Zhu, Oxland, and Wilson (2006), who stressed the importance of calibration to minimise the system errors.

A number of studies have evaluated the effect of the calibration procedure on the accuracy of the system. The proprietary software has two built-in calibration functions, (i) one-point linear and (ii) two-point power calibrations, both with an assumption that zero force equals zero output. These calibrations are conducted by applying a known uniform load to the entire previously equilibrated sensor (Tekscan, 2006). Wilson et al. (2006) and Wilson, Apreleva, Eichler, and Harrold (2003) found that measurements made using a linear calibration were more repeatable and accurate than those made with a two-point power calibration, however, studies conducted by Brimacombe, Anglin, Hodgson, and Wilson (2005) contradicted this finding and showed that the power calibration of the sensors gave significantly lower errors of $2.7 \%$, in comparison to $24.4 \%$ and $10.5 \%$ obtained for two linear calibrations conducted at $20 \%$ and $80 \%$ of the maximum load, respectively. Further, their study developed userdefined 3-point quadratic and 10-point cubic calibrations, which were found to further reduce the errors associated with the power calibration to $1.5 \%$ and $0.6 \%$, respectively. Similar results were found by DeMarco, Rust, and Bachus (2000). These studies, however, conducted the evaluation of sensor entire output without any consideration given to the output of individual sensing elements.

The previous studies evaluating sensor performance point out the importance of the appropriate calibration of the sensors in order to reduce the uncertainties in the results. This study evaluates the proprietary built-in Tekscan calibration and development of a novel polynomial 'per sensel' calibration and its ability to reduce the errors associated with the pressure determination of individual elements. In order to do so, the following methodology was established:

(i) The design and construction of a novel pressure calibration device,

(ii) The evaluation of the Tekscan proprietary calibration,

(iii)The development and evaluation of a calibration procedure for each sensel with 10 predetermined pressures applied over the operating range where the non- 
responsive sensels were disregarded; referred in the following as 'multi-point per sensel calibration with sensel selection', and

(iv) The correction of the multi-point per sensel calibration with sensel selection.

This was conducted in order to determine an effective method to measure the pressure distribution below pneumatic agricultural tyres on both hard surfaces and within the soil profile (Misiewicz, 2010).

\section{The design and construction of a novel pressure calibration chamber}

Each Tekscan sensor needs to be equilibrated and calibrated before being used for pressure measurements; five Tekscan sensors were selected for this study, equilibrated and calibrated using a purpose-built pressure calibration chamber. The calibration of the sensors was conducted by two methods; firstly, the sensors were equilibrated and calibrated following the guidelines from Tekscan (Tekscan, 2006). The second method involved the development of a novel calibration procedure where each sensing element was calibrated separately using the multi-point data procedure. An evaluation of the accuracy of the sensors was conducted after the sensors were calibrated and equilibrated.

The following Tekscan sensors, shown in Fig. 1, were selected, as their size, shape and pressure range were the most suitable for the tyre contact pressure study by Misiewicz (2010):

- Conformat system: Model 5330 sensor

- standard pressure range: $0-0.55 \times 10^{5} \mathrm{~Pa}$

- sensor dimensions: $471.4 \mathrm{~mm}$ x $471.4 \mathrm{~mm}$

- number of sensing elements: 1024

- I-Scan system: Model 6300-A and 6300-B sensors

- $\quad$ standard pressure range: $0-3.45 \times 10^{5} \mathrm{~Pa}$

- sensor dimensions: $264.2 \mathrm{~mm}$ x $33.5 \mathrm{~mm}$

- number of sensing elements: 2288

- I-Scan system: Model 9830-A and 9830-B sensors

- standard pressure range: $0-0.7 \times 10^{5} \mathrm{~Pa}$

- $\quad$ sensor dimensions: $188.6 \mathrm{~mm}$ x $203.2 \mathrm{~mm}$

- number of sensing elements: 176 
The standard pressure range of each sensor can be increased or decreased by a factor of 10 using the appropriate software scaling function.

In order to provide a fundamental and independent calibration of the Tekscan sensors, a calibration chamber was designed and constructed to allow the application of uniform pneumatic pressure to all sensing elements being simultaneously calibrated (Misiewicz, 2010). The calibration system consisted of a lower and upper plate, as shown in Figure-Fig. 2 and Figure-Fig. 3. A Tekscan sensor was placed on the smooth ground upper surface of the bottom plate and then a diaphragm placed on the sensor followed by the top plate. The two plates were bolted together by 28 M16 set-screws. Pressure was applied inside the device from the top into the plenum chamber and recorded using a digital pressure gauge (range of 0 $-20 \times 10^{5} \mathrm{~Pa}$ ). The system was designed for a maximum safe working pressure of $34.5 \times 10^{5}$ Pa. Air can be used to pressurise the device up to $8 \times 10^{5} \mathrm{~Pa}$, whilst oil is recommended for pressures above $8 \times 10^{5} \mathrm{~Pa}$. Depending on the pressure range, a flexible rubber or polythene membrane was used as the diaphragm to seal the device whilst allowing a uniform pressure application to the entire sensor. The entire system weighed $0.28 \mathrm{t}$.

\section{Evaluation of the Tekscan proprietary calibration}

Following the manufacturer's recommendations to reduce the effect of drift and hysteresis (Tekscan, 2006), each sensor was conditioned by repeatedly applying air pressure five times, before it was calibrated. Sensors were loaded with uniform pressure to values approximately $20 \%$ greater than those expected during the studies. For the equilibration and calibration air pressure was applied to the sensor as follows:

1) The equilibration was conducted in 10 increments when pressure was increased. Prior to this process a minimum pressure of $0.1 \times 10^{5} \mathrm{~Pa}$ was applied to the sensor for one minute to establish an equilibrium condition.

2) During the calibration process, a scale factor established during the equilibration process was applied by the proprietary software to each sensing element to make the output uniform between sensels. A two-point calibration was performed by applying two different pressures to the sensor ( $20 \%$ and $80 \%$ of the expected maximum pressure). The pressures were applied for one second to allow the pressure to stabilise. Using these data a power law interpolation for overall sensor based on zero load and the two known calibration loads was performed. 
Based on the proprietary calibration, the mean, maximum and minimum pressures were determined for each sensor and compared to the applied pressures measured by the air pressure gauge, as shown in Table 1. The bias errors of the overall sensel pressures were less than $3.0 \%$ for the Conformat 5300, I-Scan 6300-A and 6300-B sensors; the I-Scan 9830-A and $9830-\mathrm{B}$ produced bias errors as high as $12.5 \%$.

Figure-Fig. 4 presents a series of histograms of the residual errors obtained when the sensors were pressurised with uniform pressure. Each histogram presents all the errors obtained for the sensels of the sensor tested at the range of applied pressures. Several outliers were found for each sensor, which give evidence of the presence of "erroneous" sensels. The histograms show that the I-Scan 6300-A, 6300-B, 9830-A and 9830-B gave residual errors up to $\pm 30 \%$ nearly normally distributed around " 0 ". The Conformat 5330 was found to have a tendency to record a higher-than-applied pressure with the errors below $10 \%$. This illustrates that the Tekscan sensors calibrated using the proprietary software give acceptable errors of the mean pressure with some sensels giving large variations in the pressure distribution up to $30 \%$.

As shown by Misiewicz (2010), the entire area of Conformat 5330 provided errors below $10 \%$, and $98 \%$ of the area gave errors less than $5 \%$. However, the other four sensors were generally associated with larger errors and only $92 \%-98 \%$ of the sensing area gave errors less than a $10 \%$ error, and $64 \%-86 \%$ of the area had errors less than $5 \%$.

Following calibration and equilibration using the Tekscan calibration procedure experiments involving rolling loaded tyres over the sensors on a hard surface were conducted. The data were collected by the two I-Scan 9830 sensors, which overlapped the tyre centre line by 50 $\mathrm{mm}$. Figure 5 illustrates contact pressure profiles (cross-sections) found below the centre of a smooth (with the tread removed) Trelleborg T421 Twin Implement 600/55-26.5 tyre. The raw outputs collected by the two sensors from the overlapping area, plotted in Fig. 5a, were found to be similar. When the Tekscan proprietary calibration and equilibration were applied to the data, the results were found to differ significantly by up to $26 \%$ (Fig.tre 5b). Hence, the results shown in Fig. 5 confirm a requirement for an evaluation of data modification protocols associated with the proprietary calibration and equilibration, and a requirement for an improved calibration protocol. 
To understand the raw output (non-calibrated and non-equilibrated) and the functions that are applied to the data by the Tekscan software, the raw data were collected and analysed. As the Tekscan calibration procedure involves establishing one regression curve for an entire sensor, which is an average value for all the sensing elements, it was necessary to verify the raw output data of each individual sensel in order to determine if they had similar characteristics.

In order to do this the sensors were placed in the calibration chamber and air pressure was applied. Both, the raw output data (non-calibrated and non-equilibrated) and equilibrated data recorded, were plotted against the applied pressure, as shown for the I-Scan 9830-A sensor in Fig. 6. The data were plotted using the proprietary convention for calibration, to enable the pressure to be readily determined from the Tekscan output in the form of the equations given. Figure 6 shows how the Tekscan equilibration function modifies the results. Plotting the data has verified that the output characteristic varied between the sensels, however, the equilibration procedure was found to account for the different calibration characteristics to a great extent. Best-fit power functions were established to visualise the differences in the sensor performance. After the equilibration was applied to the raw output, the maximum variation was found to decrease from $130 \%$ to $6 \%$. This agrees with findings of Maurer et al. (2003), who proved that sensor equilibration, which accounts for variations between the individual sensing elements of a sensor, is effective in reducing inter-cell variations.

The evaluation of the raw data showed the variations between the individual sensing elements of a sensor and the importance of equilibration in reducing these variations. This confirmed a need for a multi-point calibration of all the sensors and a separate consideration of each sensing element during the calibration to account for the equilibration of sensors.

\section{The development and evaluation of the multi-point per sensel calibration with} sensel selection

The second method of calibrating the sensors involved directly recording the raw values available from the Tekscan system when applying a number of air pressures to the sensels in increasing increments. This was conducted in order to establish a multi-point calibration for each individual sensing element and to locate the sensors giving no output or values that were in excess of the expected range. 
Before calibrating the sensors, they were conditioned by repeatedly (x5) applying a uniform pressure to values approximately $20 \%$ greater than those expected during the tests. Then the multi-point calibration was conducted, this involved an application of air pressure across the sensor in 10 increasing increments from $10 \%$ to $100 \%$ of the maximum pressure expected for each sensor. Each pressure was applied for one second and the raw data recorded and processed in order to establish linear, power, second, third and fourth order polynomial relationships. They were then used for the evaluation of the multi-point per sensel calibration. The identification of erroneous and non-responsive sensels was required in order to eliminate them before the calibration constants were applied. The de-selection was based on the following criteria:

- non-responsive sensels: the sensels giving zero output when loaded,

- erroneous sensels: visual selection of outliers.

The data obtained for the 9830-A sensor were selected for evaluation of the multi-point per sensel calibration, as this sensor was the most appropriate for the experimental work of Misiewicz (2010). The residual errors were plotted as histograms for each type of regression curve and are shown in Fig. 7. The results showed that the design of the multi-point per sensel calibration significantly improved the accuracy of the pressure measurements by reducing the bias errors below $1 \%$. The residual errors were found to be below $7 \%$ for the linear calibration, below $5 \%$ for the $2^{\text {nd }}$ order polynomial calibration and below $4 \%$ for the $3^{\text {rd }}$ and $4^{\text {th }}$ order polynomials. The power function was found to have the least effect in reducing the errors, as the residuals were found to vary from $-10 \%$ to $+20 \%$. Therefore, the findings confirmed that the polynomial functions give the closest fit to the data and improve the accuracy of the system.

As shown by Misiewicz (2010), the polynomial regression curves gave the best accuracy of the data for the $9830-\mathrm{A}$ sensor with the $4^{\text {th }}$ order polynomial providing residual errors below $3 \%$ for all sensing elements of the sensor and $88 \%$ of the elements giving errors below $1 \%$. In the case of the linear regression, $99 \%$ of the sensor area provided errors below $5 \%$ and only $51 \%$ was associated with errors less than $1 \%$. The power function provided the greatest residual errors, with $71 \%$ of the area having errors less than $3 \%$ and only $32 \%$ of the area had errors less than $1 \%$. 
In order to further check the accuracy of the multi-point calibration, sets of raw data were obtained by loading the 9830 -A sensor with air pressure in the calibration chamber with a previously established multi-point calibration applied to the data. The statistical errors of individual sensing elements were calculated and presented in Fig. 8. Generally, the results were found to slightly underestimate the pressures and the highest statistical errors were found again for the power function, which varied from $-10 \%$ to $+3 \%$. For the linear relationships the errors varied from $-7 \%$ and $+3 \%$. For the $2^{\text {nd }}, 3^{\text {rd }}$ and $4^{\text {th }}$ order polynomials the errors were the smallest, varying between $-3 \%$ and $+2 \%$.

The polynomial models give the largest amount of sensing area of the 9830- sensor with small errors; for the $2^{\text {nd }}$ and $3^{\text {rd }}$ order polynomial almost $100 \%$ of the sensor area was associated with statistical errors lower than $3 \%$ and $60 \%$ of the area had errors lower than $1 \%$. The $4^{\text {th }}$ order polynomial function gave slightly improved results as $100 \%$ and $67 \%$ of the sensing area had statistical errors lower than $3 \%$ and $1 \%$, respectively, while for the linear and power functions only $32 \%$ and $30 \%$ of the area gave errors smaller than $1 \%$, and $80 \%$ and $60 \%$ gave errors smaller than $3 \%$ (Misiewicz, 2010).

The evaluation of the performance of sensors calibrated using the multi-point per sensel calibration with sensel selection was found to improve the accuracy of the results (below $\underline{ \pm+}$ $4 \%$ ), although there were still some residual variations but they were lower than the variations obtained following the proprietary recommended calibration (up to $\pm 30 \%$ ).

\section{The correction of the multi-point per sensel calibration with sensel selection}

Tekscan sensors have a varied output that depends on the materials used to apply the pressure to the sensor (Tekscan, 2006). The sensors consist of active and non-active areas and the load applied to the active area of each sensel is measured. An assumption made regarding the system is that the same load is applied to the non-active area and the system determines the pressure as the total load over the sensel area. Hence, the flexibility of the material that is in contact with the sensor plays an important role in pressure transfer. It can be assumed that for the highest levels of accuracy, Tekscan sensors should be calibrated with exactly the same interface material as the one used during testing. Unfortunately this is not always possible. In this study, during the calibration, a sensor was placed on the smooth ground surface of a steel plate; a flexible rubber or polythene diaphragm was then placed over the sensor. Air pressure was uniformly applied to the diaphragm. In the tyre contact pressure study of 
Misiewicz (2010), both, the hard surface and soil experiments, involved a smooth aluminium plate loaded by a pneumatic tyre and Tekscan sensor placed at the interface either directly or through the soil. Materials with similar characteristics were used in both the calibration and experiments. The rubber and polythene membrane, used in the calibration process, were expected to distribute the pressure in a manner similar to a pneumatic tyre. This was evaluated by comparing the total load applied to the tested tyres and the total load recorded by Tekscan sensors. In case of a poor agreement, a correction factor would need to be developed to account for the compliance of different interface materials and to enable the system to provide pressure measurements between different surface interfaces.

In order to evaluate the requirement for a correction factor, two sets of experiments were conducted. These were as follows:

a. A comparison of the calibration and test environments in a small scale controlled $\underline{\text { study }}$

This was conducted using the I-Scan 9830 sensors as they were selected, as being those that might produce the greatest discrepancy due to a relatively low spatial resolution of sensels (active area of each sensel: $6.3 \mathrm{~mm}$ x $3.8 \mathrm{~mm}$ ). Initially a multi-point per sensel calibration with the de-selection of faulty sensels was conducted, which was based on the data obtained when loading the sensors in the pressure calibration chamber. The following experiments were then conducted:

- The sensors were loaded with a number of uniform pressures in the pressure calibration chamber (with a polythene diaphragm).

- In order to simulate the hard surface tyre loading environment, the sensors were covered with a polythene membrane and a number of individual sensing elements were randomly selected (excluding any faulty sensels) to which a range of $(0-500 \mathrm{~g})$ laboratory weights were individually applied through a $2 \mathrm{~mm}$ thick square rubber pad of the size of the sensor active area (Fig. 9, left and middle).

- To simulate the soil conditions, the small rubber pad was replaced with sandy loam soil confined in a $2 \mathrm{~mm}$ thick larger rubber pad with a central square of the same dimensions as the active area of the sensel removed. Then a range of $(0-500 \mathrm{~g})$ laboratory weights was applied to the soil placed on the selected sensels (Fig. 9, right). 
The effect of the loads applied to the sensels using the three different media (polythene diaphragm, rubber pad and soil) were recorded and compared, as shown in Fig. 10 and Fig. 11. The figures present data obtained for one random sensing element, as other randomly selected sensels showed similar relationships. The tests conducted in the pressure calibration chamber, using polythene diaphragm, provided data recorded by Tekscan that agree with the applied values (Fig. 10), which confirms that the data obtained when loading the sensor in the pressure calibration chamber agree with the previous calibration conducted using the same device. The relationships between the applied and recorded load, shown in Fig. 11, were found to be linear, however, the data recorded by Tekscan, when the loads were applied through the rubber pad and soil, were found to be lower than the applied load. The slopes of the relationships between the applied and measured load were found to be 0.534 and 0.567 for the rubber pad and soil block, respectively. The dissimilarity is related to differences in interface material used and proved a requirement for a correction factor to be used for contact pressure tests if they were conducted using the I-Scan 9830 sensors.

\section{b. A comparison of the load applied to tyres and recorded by the Tekscan system} In order to check similarity of the compliance factor during the calibration and experiments, a comparison of the weight computed from the Tekscan vertical pressure distribution and the total weight applied to a tyre, obtained by Misiewicz (2010), for the two types of Tekscan sensors was conducted.

\section{i. $\quad$ I-Scan 9830 sensors}

Figure-Fig. 12 presents relationships of the applied and recorded load for the tyre tested on, both, the hard surface and the soil using the I-Scan 9830 sensors. The recorded loads were less than the applied loads. The slope of the relationship between the applied and recorded load was found to be 0.639 and 0.553 on hard surface and in the soil, respectively, which was similar to the results obtained in the small scale controlled study.

\section{ii. $\quad$ I-Scan 6300 sensors}

The I-San 6300 sensors have a higher spatial resolution (active area of each sensel: $3.2 \mathrm{~mm} \mathrm{x}$ $2.0 \mathrm{~mm}$ ) than the I-Scan 9830 sensors. The comparison of the loads applied to the tyres and measured by Tekscan, when testing agricultural tyres using the 6300 sensors, agreed to within $\pm 10 \%$ of the overall slope of the relationship of 0.95 , as illustrated in Fig. 13 . 
The comparison of the load applied to tyres and measured by Tekscan sensors showed that there is a difference between the applied loads and recorded values obtained for the I-Scan 9830 sensors. This difference was not found to be significant for the 6300 sensor, which has a higher spatial resolution. Therefore, this discrepancy found for the 9830 sensors was assumed to be caused by the fact that different loading materials were used for the calibration and pressure measurements. When the sensors are pressurised with air during the calibration, the pressure is uniform as the air follows the shape of Tekscan sensors. However, soil and rubber are less deformable and follow the shape of the sensors less well. As the recorded loads were considerably lower than the loads applied, it indicates that a large part of the load applied concentrated on the non-active areas of the sensors.

In order to correct the performance of Tekscan sensors in determining the contact pressure between materials different to those used in sensor calibration, all individual contact pressure data points obtained using the sensors should be increased by a correction factor calculated as applied load/recorded load for each test. This adjustment will lead to an agreement between the Tekscan recorded load and the load applied to the sensor.

Finally, the performance of Tekscan sensors in contact pressure measurements below agricultural tyres was evaluated by using the sensors for the contact pressure determination below a selection of tyres. Figure-Fig. 14 presents the contact pressure profile obtained below the treadless T421 Twin Implement 600/55-26.5 tyre after the novel multi-point per sensel calibration was applied to the raw data, previously shown in Figure-Fig. 5. A close agreement between the overlapping sensels in the centre of the tyre contact area was found. This indicates that the development of the new calibration procedure resulted in a significant improvement of the accuracy of the sensors and made it possible to use them to determine the pressure distribution below tyres.

\section{Figure Fig. 15 shows an example of tyre contact pressure distribution of a Goodyear} 11.50/80-15.3 implement tyre on a hard surface at its recommended load of 2.18 tenne at 4.1 x $10^{5} \mathrm{~Pa}$ inflation pressure. It was obtained using sensors which were previously calibrated using the multi-point per sensel calibration with sensel selection. It is recommended that this calibration procedure is used to evaluate the accuracy of the other available pressure mapping systems. 


\section{Conclusions}

1. A pressure calibration chamber has shown to be a valuable tool to calibrate the sensors and to evaluate the pressure distribution of the sensors.

2. The pressure mapping sensors calibrated with the proprietary built-in calibration give the majority of bias errors below $3 \%$ and the maximum error of $12.5 \%$ when measuring the mean pressure, however, individual sensel errors of $\pm++30 \%$ were found to be present.

3. When using the multi-point per sensel calibration with sensel selection the bias errors have been reduced below $1 \%$ with both residual and statistical errors of $\pm 4 \%$ for the polynomial relationships.

4. The sensor equilibration has been found to decrease the maximum variations of Tekscan output from $130 \%$ to $6 \%$.

5. Correction to the Tekscan output is required if the sensors are used to measure pressure between various interfaces different from those used in the calibration procedure. The compliance factor can be calculated as a ratio of applied load to load recorded by the sensor.

6. Tyre contact pressure distribution can be more confidently determined using Tekscan sensors after they are calibrated using the multi-point per sensel calibration with sensel selection, a new calibration procedure which improves the accuracy of the sensors.

\section{References}

Brimacombe, J., Anglin C., Hodgson A., \& Wilson D. (2005). Validation of calibration techniques for Tekscan pressure sensors. Proceedings of the 20th Congress of the International Society of Biomechanics, Cleveland, OH, 31 July - 5 August 2005.

DeMarco, A.L., Rust, D.A., \& Bachus, K.N. (2000). Measuring contact pressure and contact area in orthopedic applications: Fuji Film vs. Tekscan. $46^{\text {th }}$ Annual Meeting of Orthopedic Research Society, Orlando, Florida, 12 - 15 March 2000.

Drewniak, E.I., Crisco, J.J., Spenciner, D.B., \& Fleming, B.C. (2007). Accuracy of circular contact area measurements with thin-film pressure sensors. Journal of Biomechanics, 40, $2569-2572$.

Ferguson-Pell, M., Hagisawa, S., \& Bain, D. (2000). Evaluation of a Sensor for Low Interface Pressure Applications. Medical Engineering \& Physics, 22, no. 9, 657 - 663.

Horn, R., Fleige, H., Peth, S., \& Peng, X. (2006). Soil management for sustainability, Advances in GeoEcology. The 17th Triennial International Soil Tillage Research Conference, CATENA VERLAG GMBH, Kiel, Germany, 28 August - 3 September 2006. 
Maurer, J.R., Ronsky, J., Loitz-Ramage, B., Andersen, M., Zernicke, R., \& Harder, J. (2003). Prosthetic socket interface pressures: Customized calibration technique for the TEKSCAN Fsocket system. Summer Bioengineering Conference, Florida, 25 - 29 June 2003.

Misiewicz, P.A. (2010). The evaluation of the soil pressure distribution and carcass stiffness resulting from pneumatic agricultural tyres, $\mathrm{PhD}$ Thesis, Cranfield University.

Soane, B.D., \& Ouwerkerk, C. (1994). Soil compaction problems in word agriculture. In B. D. Soane, \& C. Ouwerkerk (Eds). Soil compaction in crop production (pp. 1-21). The Netherlands: Elsevier Science.

Sumiya, T., Suzuki, Y., Kasahara, T., \& Ogata, H. (1998). Sensing stability and dynamic response of the F-scan in-shoe sensing system: A technical note. Journal of Rehab. Res. Devel., 35, $192-200$.

Tekscan. (2006). Tekscan I-Scan User Manual, Tactile force and pressure measurements system, v. 5.8x, published by Tekscan Inc, South Boston, Mass., USA.

Tekscan. (not dated a). Tekscan home page, available at: www.tekscan.com (accessed on 15 June 2013).

Tekscan (not dated b). Tekscan technology, available at: http://www.tekscan.com/tekscantechnology (accessed on 1 April 2014).

Wilson, D.C., Niosi, C.A., Zhu, Q.A,. Oxland T.R., \& Wilson, D.R. (2006). Accuracy and repeatability of a new method for measuring facet loads in the lumbar spine. Journal of Biomechanics, 39, 348 - 353.

Wilson, D.R., Apreleva, M.V., Eichler, M.J., \& Harrold, F.R. (2003). Accuracy and repeatability of a pressure measurement system in the patellofemoral joint. Journal of Biomechanics, 36, 1909 - 1915. 


\section{Figures:}

Fig. 1 - Tekscan pressure mapping sensors (from left: Conformat model 5330 sensor, I-Scan model 6300 sensors, I-Scan model 9830 sensors)

Fig. 2 - Pressure calibration chamber

Fig. 3 - Cross section of the pressure calibration chamber showing the individual components Fig. 4 - Residual error histograms for the 5 Tekscan sensors calibrated using the Tekscan proprietary calibration (please note vertical scales are different between the five sub-figures) Fig. 5 - Cross sectional profiles of tyre contact pressure obtained below a smooth Trelleborg T421 Twin Implement 600/55-26.5 tyre using I-Scan 9830 sensors; a: non-calibrated and non-equilibrated data; $b$ : data calibrated and equilibrated following Tekscan procedure. Dashed ovals indicate the results obtained by overlapping sensels

Fig. 6 - Pressure applied vs. output for each sensing element of the I-Scan 9830-A sensor (top: non-calibrated and non-equilibrated data, bottom: non-calibrated but equilibrated data) Fig. 7 - Residual errors for the I-Scan 9830-A sensor after multi-point per sensel calibration; a: linear, b: power, c: $2^{\text {nd }}, d$ : $3^{\text {rd }}$ and e: $4^{\text {th }}$ order polynomial (please note horizontal and vertical scales are different between the five sub-figures)

Fig. 8 - Statistical errors for I-Scan 9830-A sensor after the multi-point per sensel calibration; a: linear, b: power, c: $2^{\text {nd }}, \mathrm{d}: 3^{\text {rd }}$ and e: $4^{\text {th }}$ order polynomial (please note horizontal and vertical scales are different between the five sub-figures)

Fig. 9 - Small scale controlled study on the I-Scan 9830 sensors (left and middle: rubber pad tests, right: soil test)

Fig. 10 - Measured vs. applied load for I-Scan 9830-A sensor loaded in the pressure calibration chamber using a polythene diaphragm

Fig. 11 - Measured vs. applied load for I-Scan 9830-A sensor; left: load applied through a rubber pad, right: load applied through soil (1:1 line dashed)

Fig. 12 - Measured vs. applied load for I-Scan 9830 sensors when loaded by the T421 Twin Implement 600/55-26.5 tyre; left: hard surface, right: soil (1:1 line dashed)

Fig. 13 - Measured vs. applied load for I-Scan 6300 sensor when loaded by an 11.50/80-15.3 implement tyre on the hard surface (1:1 line dashed)

Fig. 14 - Cross sectional profile of tyre contact pressure below the treadless T421 Twin Implement 600/55-26.5 tyre obtained using I-Scan 9830 sensors; data calibrated and equilibrated according to the multi-point per sensel calibration. Dashed ovals indicate the results obtained by overlapping sensels 
498 Fig. 15 - A Goodyear 11.50/80-15.3 implement tyre at 2.18 tonne load and $4.1 \times 10^{5} \mathrm{~Pa}$

499 inflation pressure; left: tyre tread pattern; right: tyre contact pressure distribution $\left(10^{5} \mathrm{~Pa}\right)$

500 obtained using the multi-point per sensel calibration with sensel selection (direction of travel:

501 from right to left)

502

503 
505 Table:

506 Table 1 - Pressure and bias error results based on the Tekscan proprietary calibration 507 

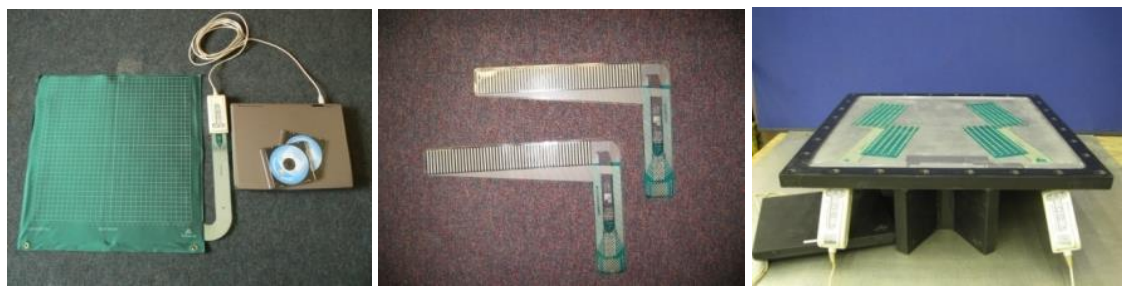

$511 \quad$ Fig. 1

512 Tekscan pressure mapping sensors (from left: Conformat model 5330 sensor, I-Scan model 513 6300 sensors, I-Scan model 9830 sensors)

514

515 
Fig. 2

519 Pressure calibration chamber 


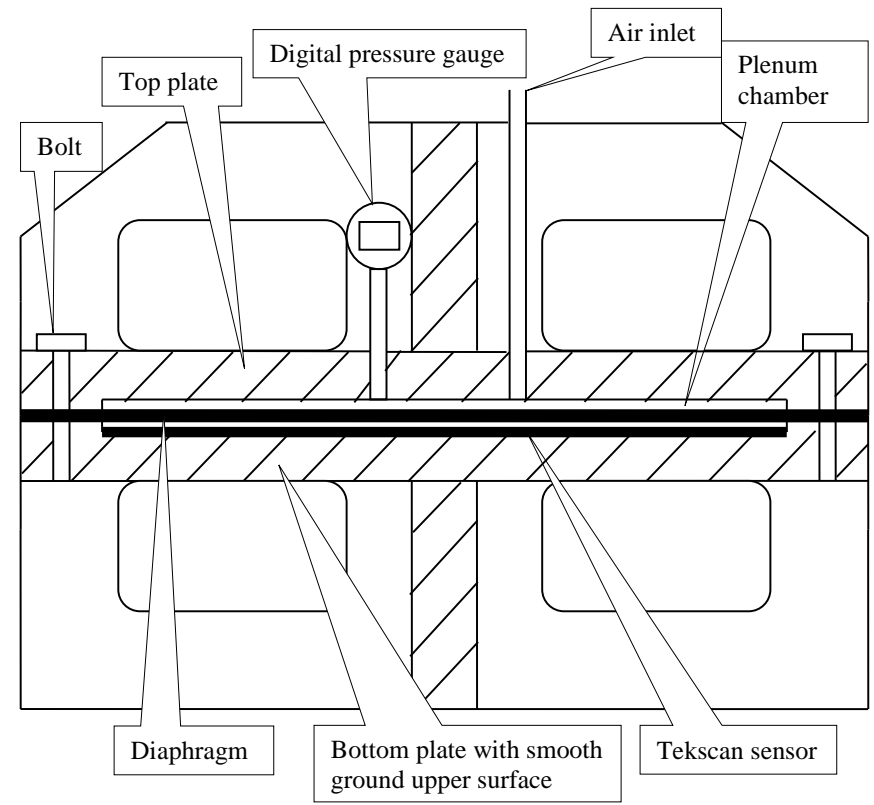

538 Cross section of the pressure calibration chamber showing the individual components 

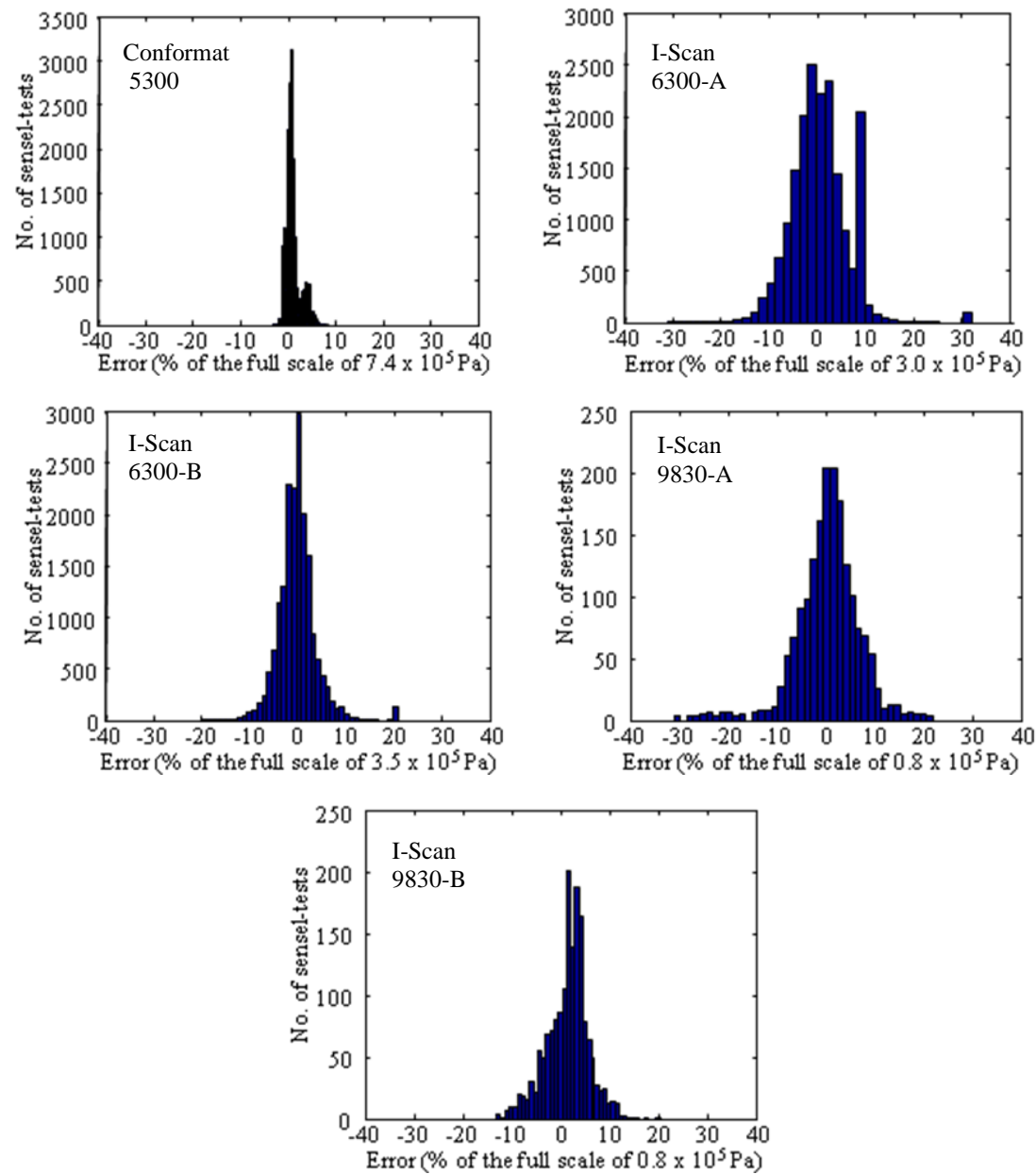

Fig. 4

Residual error histograms for the 5 Tekscan sensors calibrated using the Tekscan proprietary calibration (please note vertical scales are different between the five sub-figures) 

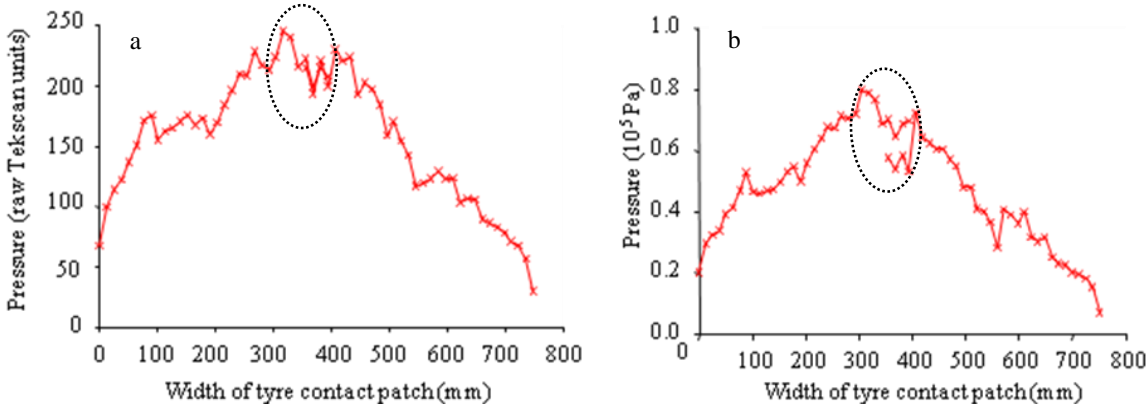

551 Fig. 5

Cross sectional profiles of tyre contact pressure obtained below a smooth Trelleborg T421

Twin Implement 600/55-26.5 tyre using I-Scan 9830 sensors; a: non-calibrated and nonequilibrated data; b: data calibrated and equilibrated following Tekscan procedure. Dashed ovals indicate the results obtained by overlapping sensels 


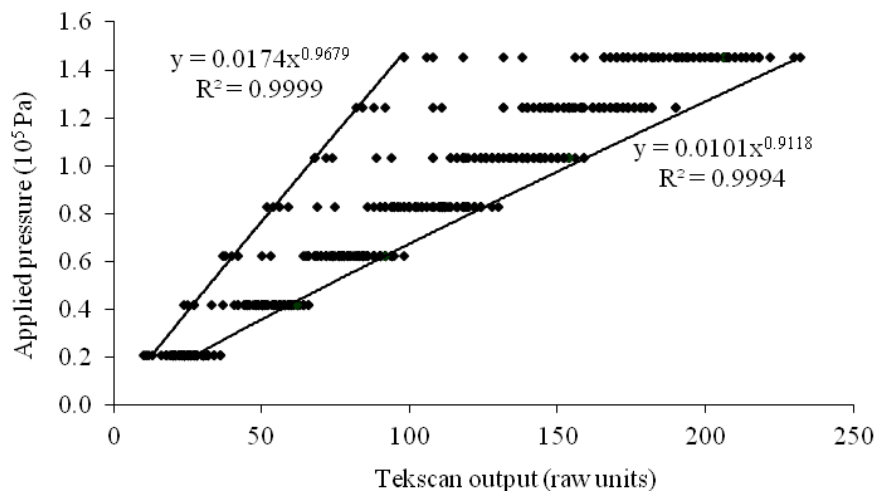

559

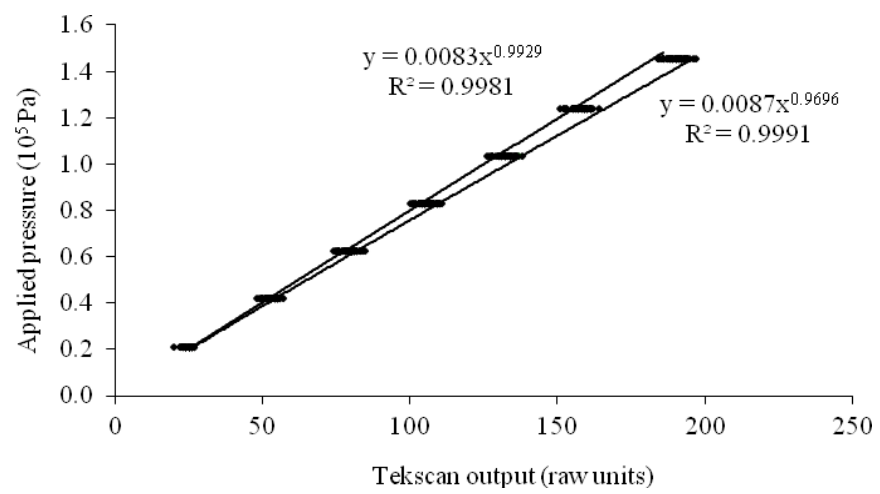

Fig. 6

Pressure applied vs. output for each sensing element of the I-Scan 9830-A sensor (top: non- 

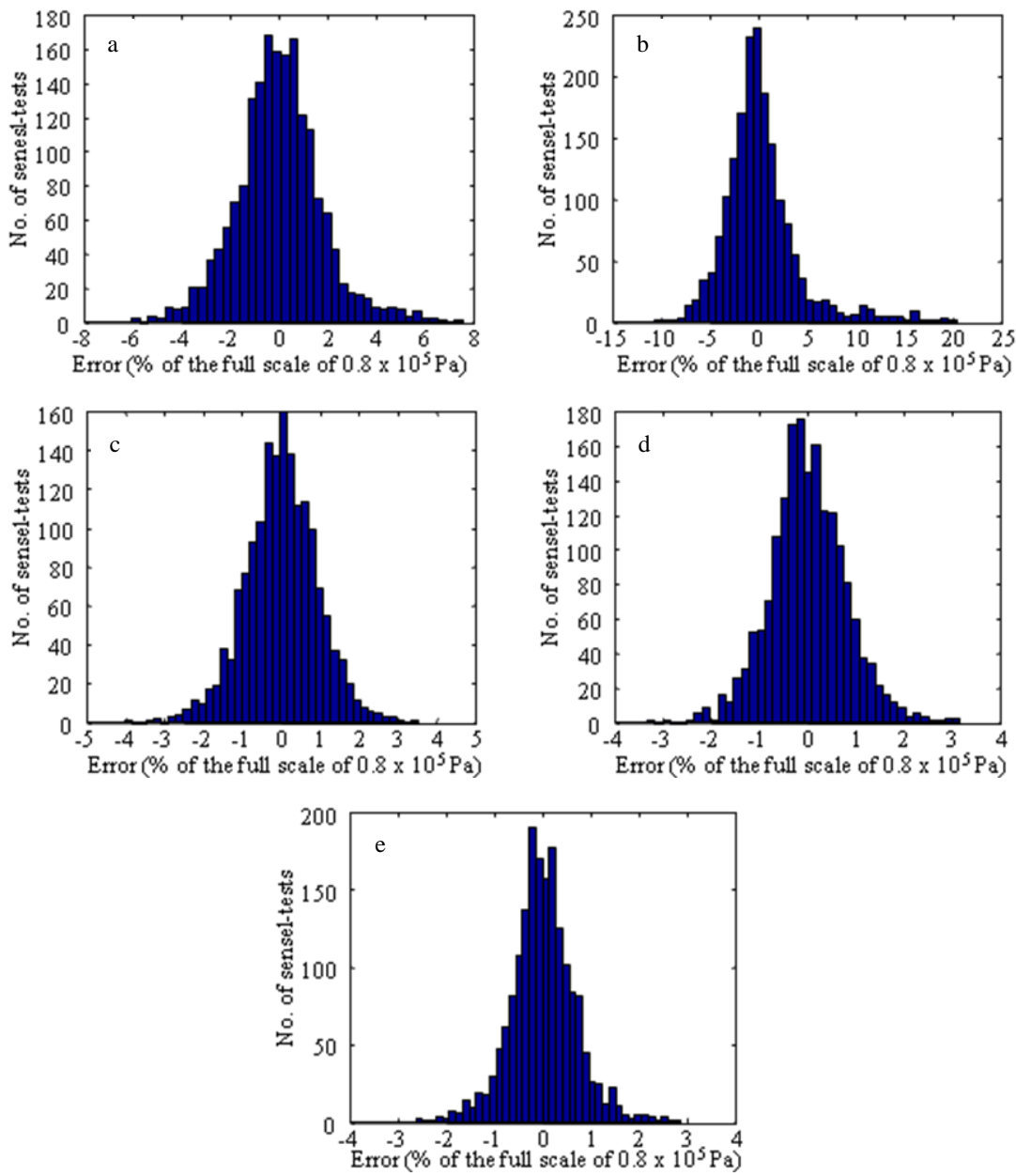

Fig. 7

Residual errors for the I-Scan 9830-A sensor after multi-point per sensel calibration; a: linear, b: power, c: $2^{\text {nd }}, d$ : $3^{\text {rd }}$ and e: $4^{\text {th }}$ order polynomial (please note horizontal and vertical scales are different between the five sub-figureFig.sfigures) 

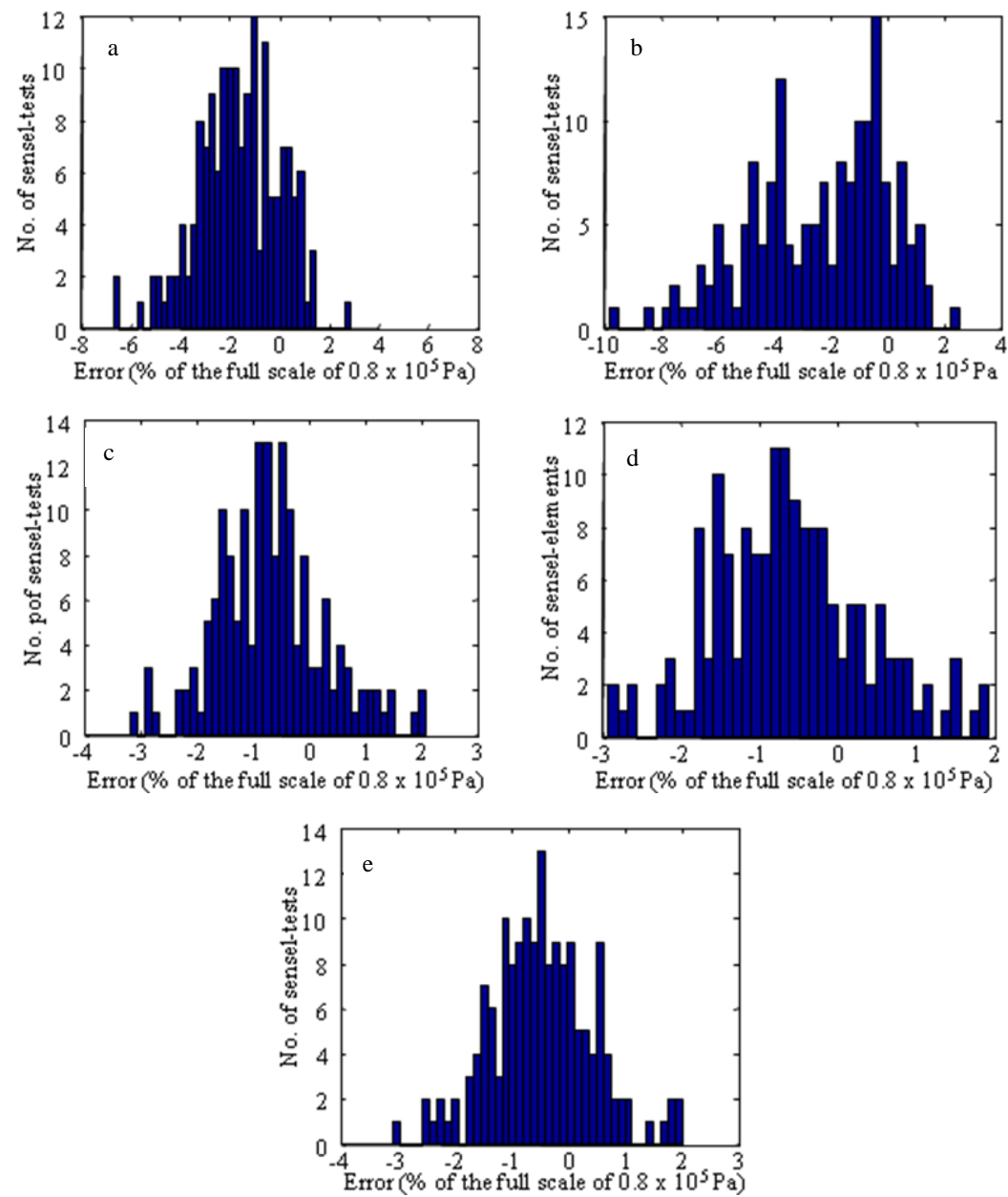

Fig. 8

Statistical errors for I-Scan 9830-A sensor after the multi-point per sensel calibration; a: linear, b: power, c: $2^{\text {nd }}, \mathrm{d}: 3^{\text {rd }}$ and e: $4^{\text {th }}$ order polynomial (please note horizontal and vertical scales are different between the five sub-figuresfigureFig.s) 


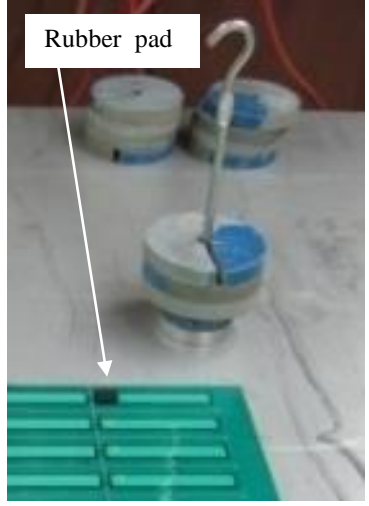

587

590

591

Fig. 9 right: soil test)
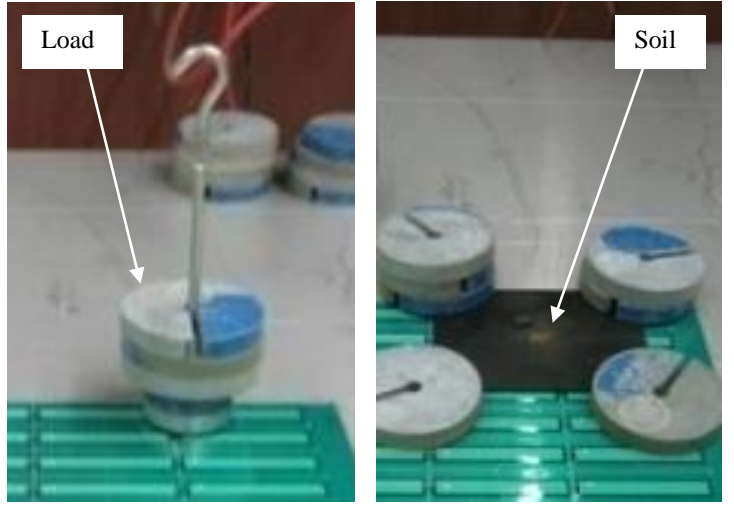

$50 \mathrm{~mm}$

Small scale controlled study on the I-Scan 9830 sensors (left and middle: rubber pad tests, 


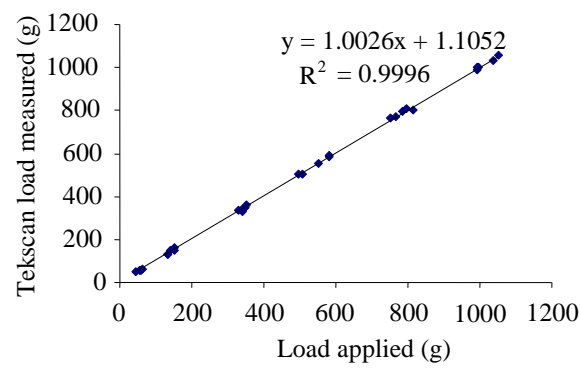

593

594 Fig. 10

595 Measured vs. applied load for I-Scan 9830-A sensor loaded in the pressure calibration

596 chamber using a polythene diaphragm

597 

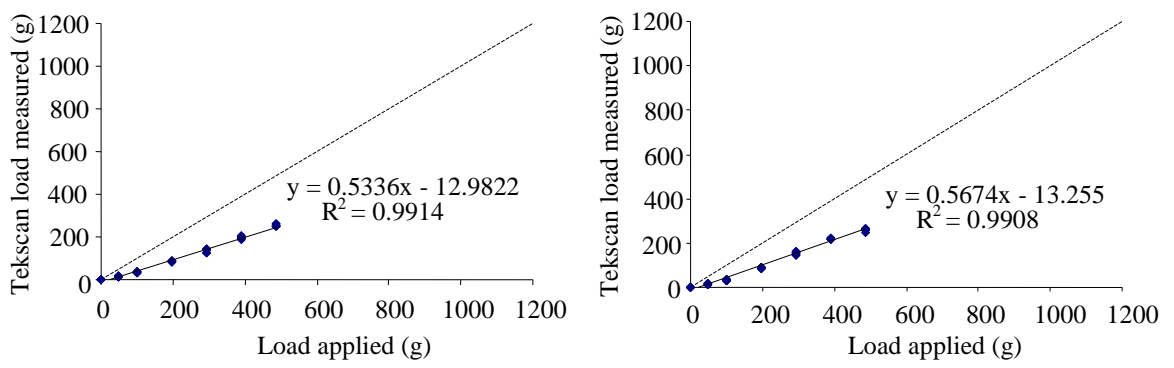

600

$601 \quad$ Fig. 11

602 Measured vs. applied load for I-Scan 9830-A sensor; left: load applied through a rubber pad, 603 right: load applied through soil (1:1 line dashed) 

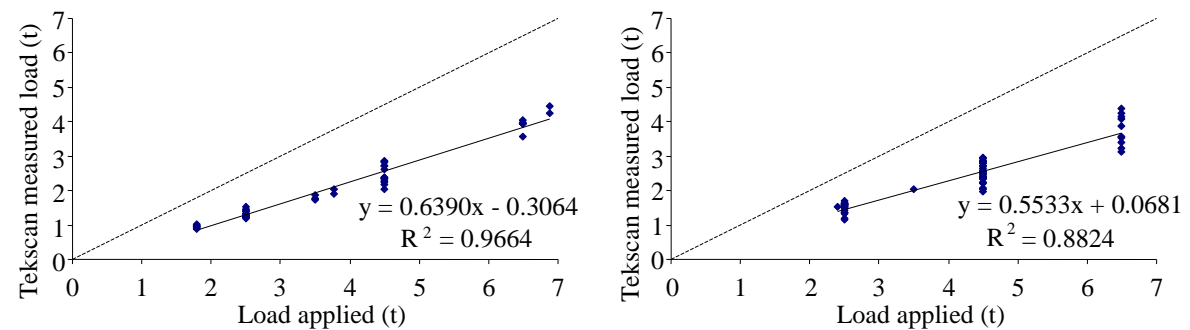

607

608 Fig. 12

609 Measured vs. applied load for I-Scan 9830 sensors when loaded by the T421 Twin Implement 610 600/55-26.5 tyre-; left: hard surface, right: soil (1:1 line dashed) 


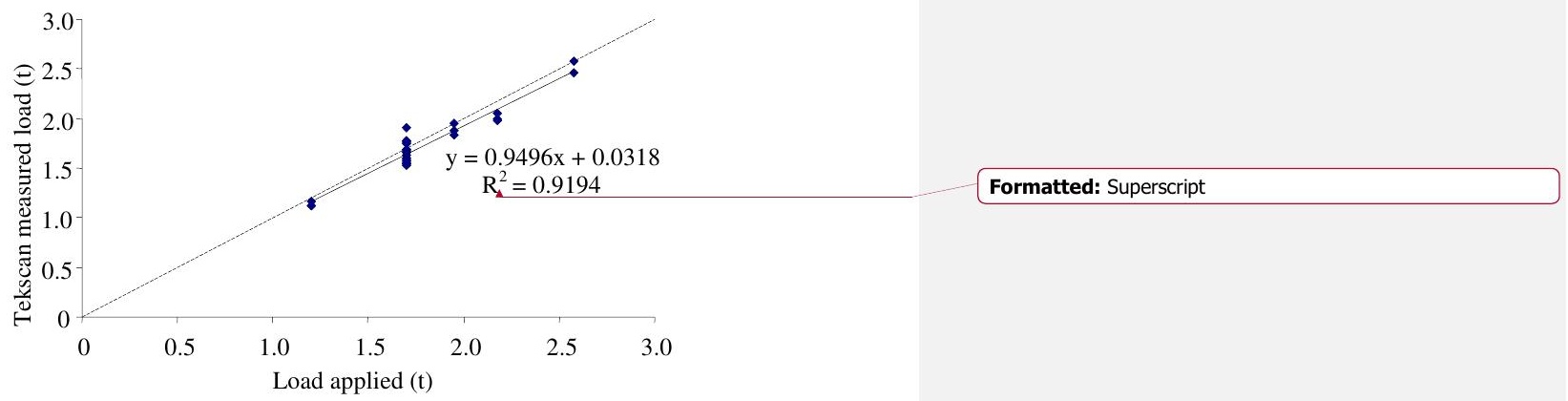

613

614 Fig. 13

615 Measured vs. applied load for I-Scan 6300 sensor when loaded by an 11.50/80-15.3

616 implement tyre on the hard surface (1:1 line dashed)

617

618 


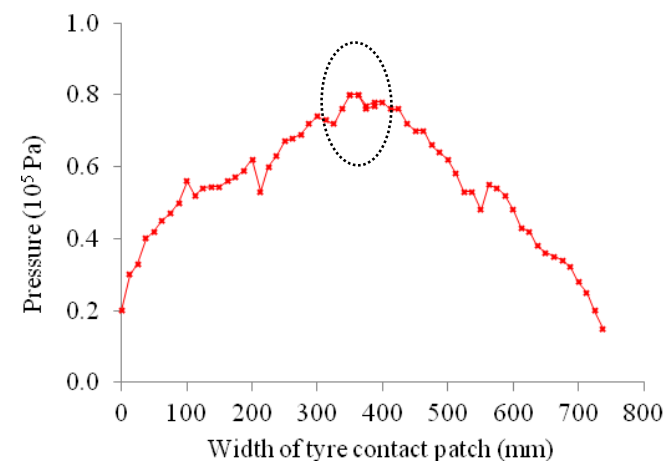

621 Fig. 14

622 Cross sectional profile of tyre contact pressure below the treadless T421 Twin Implement

623 600/55-26.5 tyre obtained using I-Scan 9830 sensors; data calibrated and equilibrated

624 according to the multi-point per sensel calibration. Dashed ovals indicate the results obtained 625 by overlapping sensels 


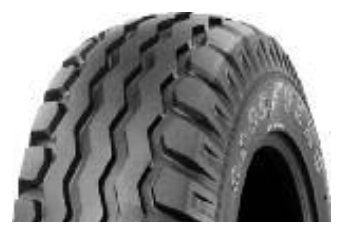

628

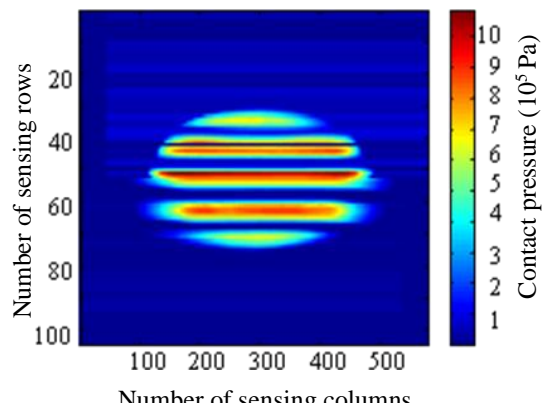

629 Fig. 15

Number of sensing columns

630 A Goodyear 11.50/80-15.3 implement tyre at 2.18 tenne load and $4.1 \times 10^{5}$ Pa inflation 631 pressure; left: tyre tread pattern; right: tyre contact pressure distribution $\left(10^{5} \mathrm{~Pa}\right)$ obtained 632 using the multi-point per sensel calibration with sensel selection (direction of travel: from 633 right to left) 
637 Table 1

638 Pressure and bias error results based on the Tekscan proprietary calibration

\begin{tabular}{|c|c|c|c|c|c|}
\hline \multirow[b]{2}{*}{ Sensor } & \multirow{2}{*}{$\begin{array}{l}\text { Pressure } \\
\text { applied } \\
\left(10^{5} \mathrm{~Pa}\right)\end{array}$} & \multicolumn{3}{|c|}{ Tekscan results } & \multirow[b]{2}{*}{$\begin{array}{c}\text { Bias error }^{1} \\
(\%)\end{array}$} \\
\hline & & $\begin{array}{c}\text { Mean } \\
\text { pressure } \\
\left(10^{5} \mathrm{~Pa}\right)\end{array}$ & $\begin{array}{c}\text { Maximum } \\
\text { pressure } \\
\left(10^{5} \mathrm{~Pa}\right)\end{array}$ & $\begin{array}{c}\text { Minimum } \\
\text { pressure } \\
\left(10^{5} \mathrm{~Pa}\right)\end{array}$ & \\
\hline \multirow{4}{*}{$\begin{array}{l}\text { Conformat } \\
5330\end{array}$} & 0.689 & 0.669 & 0.756 & 0.559 & -2.9 \\
\hline & 1.386 & 1.395 & 1.498 & 1.282 & +0.6 \\
\hline & 2.101 & 2.164 & 2.392 & 2.015 & +3.0 \\
\hline & 2.759 & 2.805 & 3.343 & 1.903 & +1.7 \\
\hline \multirow{4}{*}{$\begin{array}{l}\text { I-Scan } \\
6300-A\end{array}$} & 0.689 & 0.705 & 1.231 & 0.307 & +2.2 \\
\hline & 1.379 & 1.385 & 1.988 & 0.635 & +0.5 \\
\hline & 2.068 & 2.059 & 3.019 & 1.206 & -0.4 \\
\hline & 2.758 & 2.789 & 3.019 & 1.822 & +1.1 \\
\hline \multirow{4}{*}{$\begin{array}{l}\text { I-Scan } \\
6300-B\end{array}$} & 0.689 & 0.678 & 0.916 & 0.394 & -1.6 \\
\hline & 1.379 & 1.381 & 1.626 & 1.157 & +0.2 \\
\hline & 2.068 & 2.050 & 2.424 & 1.804 & -0.9 \\
\hline & 2.758 & 2.730 & 3.485 & 2.344 & -1.0 \\
\hline \multirow{4}{*}{$\begin{array}{l}\text { I-Scan } \\
9830-A\end{array}$} & 0.138 & 0.132 & 0.196 & 0.084 & -4.0 \\
\hline & 0.276 & 0.256 & 0.298 & 0.221 & -7.3 \\
\hline & 0.414 & 0.440 & 0.478 & 0.407 & +6.3 \\
\hline & 0.552 & 0.621 & 0.716 & 0.600 & +12.5 \\
\hline \multirow{4}{*}{$\begin{array}{l}\text { I-Scan } \\
9830-B\end{array}$} & 0.138 & 0.145 & 0.233 & 0.090 & +5.5 \\
\hline & 0.276 & 0.265 & 0.303 & 0.230 & -4.0 \\
\hline & 0.414 & 0.385 & 0.414 & 0.354 & -6.8 \\
\hline & 0.552 & 0.557 & 0.563 & 0.495 & +1.0 \\
\hline
\end{tabular}

\footnotetext{
${ }^{1}$ Bias error $(\%)$ was calculated as $100 \% \times($ Mean pressure - Pressure applied $) /($ Pressure applied).
} 